\title{
Realisation of model reference compliance control of a humanoid robot arm via integral sliding mode control
}

\author{
S. G. Khan ${ }^{1}$ and J. Jalani ${ }^{2}$ \\ ${ }^{1}$ Department of Mechanical Engineering, College of Engineering Yanbu, Taibah University, \\ Al Madinah, Saudi Arabia \\ ${ }^{2}$ Department of Electrical Engineering Technology, University Tun Hussein Onn Malaysia, \\ Batu Pahat, Malaysia \\ Correspondence to: S. G. Khan (engr_ghani@hotmail.com)
}

Received: 29 March 2015 - Revised: 26 September 2015 - Accepted: 31 October 2015 - Published: 15 January 2016

\begin{abstract}
Human safety becomes critical when robot enters the human environment. Compliant control can be used to address some safety issues in human-robot physical interaction. This paper proposes an integral sliding mode controller (ISMC) based compliance control scheme for the Bristol Robotics Laboratory's humanoid BERT II robot arm. Apart from introducing a model reference compliance controller, the ISMC scheme is aimed to deal with the robot arm dynamic model's inaccuracies and un-modelled nonlinearities. The control scheme consists of a feedback linearization (FL) and an ISMC part. In addition, a posture controller has been incorporated to employ the redundant DOF and generate human like motion. The desired level of compliance can be tuned by selecting the stiffness and damping parameters in the sliding mode variable (compliance reference model). The results show that the compliant control is feasible at different levels for BERT II in simulation and experiment. The positioning control has been satisfactorily achieved and nonlinearities and un-modelled dynamics have been successfully overcome.
\end{abstract}

\section{Introduction}

As robots are stepping into social environment, the need for more suitable and safe (compliant) control strategies are even more important (Khan et al., 2010a, b, 2014a, b; Rezoug and Hamerlain, 2014; Islam et al., 2014; Iqbal et al., 2014; Ding and Fang, 2013). Both human and robot safety is still one of the major issues in social robotics. Compliance (low stiffness) control can help addressing some safety issues (Khan et al., 2014a). There are many ways to realise compliance control schemes for human robot physical interaction. Most of the compliance control schemes are fully or partially dynamic model based. However, dynamic models are prone to inaccuracies and nonlinearities. In such cases integral sliding mode controller (ISMC) can provide a robust control solution. In addition, model reference compliance scheme can be implemented via the ISMC (Herrmann et al., 2014) more easily. Both conventional sliding mode controller (SMC) and ISMC have the following design steps (see Fig. 1):
1. reaching phase: the system state is driven from any initial state to reach the switching manifolds (the anticipated sliding modes) in finite time;

2. sliding-mode phase: the system is forced into sliding motion, $r=0$, on the switching manifolds, i.e. the switching manifold becomes an attractor.

The above two phases correspond to the following two main design steps.

1. Switching manifold selection: a set of switching manifolds are selected with prescribed desirable dynamical characteristics. Common candidates are linear hyperplanes.

2. Discontinuous control design: a discontinuous control strategy is formed to ensure the finite time reachability of the switching manifolds. The controller may be either local or global, depending upon specific control requirements. 


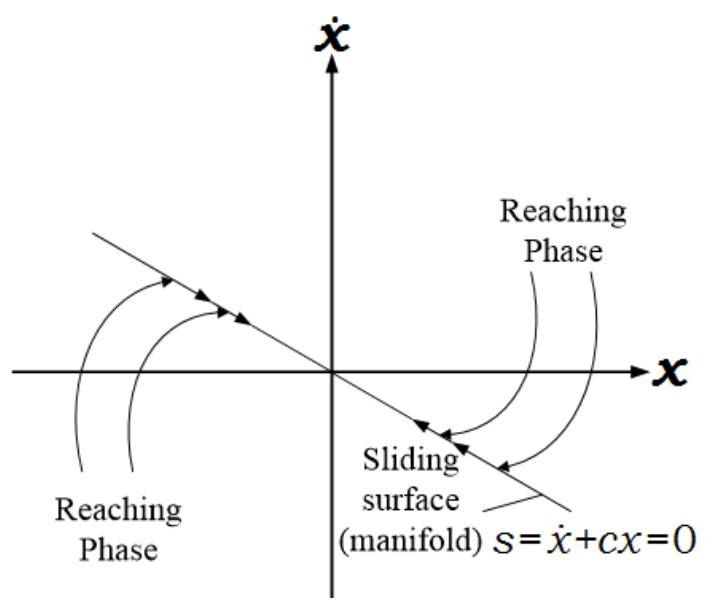

Figure 1. Sliding surface design consists of sliding phase and reaching phase.

For an ISMC, there are a few advantages outlined by Shi et al. (2008) as compared to the SMC counterpart: Sliding motion occurs right from the start of the control action, i.e. robustness (i.e. sliding mode) is guaranteed. Thus, the reaching phase is eliminated. This is not the case for the SMC. Secondly, the position control error can have large overshoots and large deviation with respect to the designed error dynamics when a conventional SMC is used in contrast to an ISMC. Lastly, the SMC control signals often have large amplitude due to chattering. Shi et al. (2008) showed these facts for a robotic system simulation. Moreover, ISMC controller design based on a perturbation estimator has significantly reduced the amplitude of chattering. Castaños and Fridman (2006) have exploited the advantages of ISMC by combining an ISMC and $H_{\infty}$-control to bring robustness to the controller against unmatched disturbances.

One of the main advantages of the ISMC and the main reason to employ it in this work is its suitability to be used as a model reference compliance controller. This will be discussed later in the paper to modify the sliding mode variable to include force feedback for compliance control.

ISMC is a well investigated control method, although practical applications are still limited, particularly in the robotics context. Some of the reasons of this may be the jittery nature and aggressiveness in the control action of sliding mode control. These type of control schemes may pose a risk of damaging the robotic devices if not implemented with care. However, due to better understanding of these schemes and availability of good control hardware these methods are getting popularity. Shi et al. (2008) have compared traditional Sliding Mode Control and ISMC through simulation for a two-link rigid manipulator. Makoto et al. (2010) have applied the ISMC to a power-assisted manipulator with a single degree of freedom through simulation and experiment. Eker and Akinal (2008) have suggested a similar scheme using an integral sliding surface in combination with the con- ventional sliding mode control. They have tested the scheme on a rather simple electro-mechanical system. In our previous work (Jalani et al., 2010a, b), an ISMC scheme for underactuated robotic fingers was simulated and experimentally tested . In this paper, experimental results of the Cartesian $(x$ and $y$ ) ISMC control in combination with a feedback linearization (FL) and a posture controller for the four degrees of freedom of the humanoid BERT II arm (see Fig. 2) are presented. ISMC has been employed here to deal with uncertainties and unmodelled nonlinearities. In addition, external force (the interaction force between robot and its environment (including human) has been included in the ISMC which results into a model reference compliance controller (Jalani et al., 2013). The dynamic model of the BERT II robot arm for the FL is obtained via MapleSim (a package of Maple) (Maplesoft, 2015).

The redundant DOF have been used to generate movement in the most human-like posture (Spiers et al., 2009a, b; Khan et al., 2010a, b; De Sapio et al., 2005). Spiers et al. (2009a, b) have implemented a robust version of the technique for a two degrees of freedom of a humanoid arm manipulator in real time. In their work, they were able to overcome a kinematic redundancy (i.e. multiple solutions produced by the robot arm manipulator) and human like movement of the arm manipulator was realized. This posture control is obtained by minimizing a gravity dependent cost function using inertia matrix of the 4 DOF dynamic model of the BERT II arm.

As mentioned before, compliance control in robotics is increasingly getting attention due to its relevance to safety in human robot interaction (HRI). Many researchers are looking into passive and active approaches to address part of safety issues in HRI (Braun et al., 2013; Potkonjak et al., 2011; Kim et al., 2012; Ficuciello and Villani, 2012; Di Natali and Valdastri, 2012; Ajwad et al., 2014, 2015). In this paper, we employ ISMC to produce compliant behaviour. A Cartesian $(X$, $Y$ and $Z$ ) model reference type of ISMC compliant scheme is simulated for 4 DOF of the humanoid BERT II robot arm ${ }^{1}$. Different compliance levels are produced by choosing spring constant and damping coefficients in the reference model (Jalani et al., 2013; Herrmann et al., 2014). The scheme will be used to address safety issues in human-robot interaction.

The contributions of the paper can be summarised as follows:

- introduction of an active compliance scheme via integral sliding mode control for a humanoid robot;

- combination of bio-inspired posture controller with ISMC scheme employing the redundancy;

- successful experimental testing of the ISMC tracking scheme on the Humanoid robot prototype.

\footnotetext{
${ }^{1}$ The mechanical design and manufacturing for the BERT II torso including hand and arm has been conducted by Elumotion (www.elumotion.com).
} 


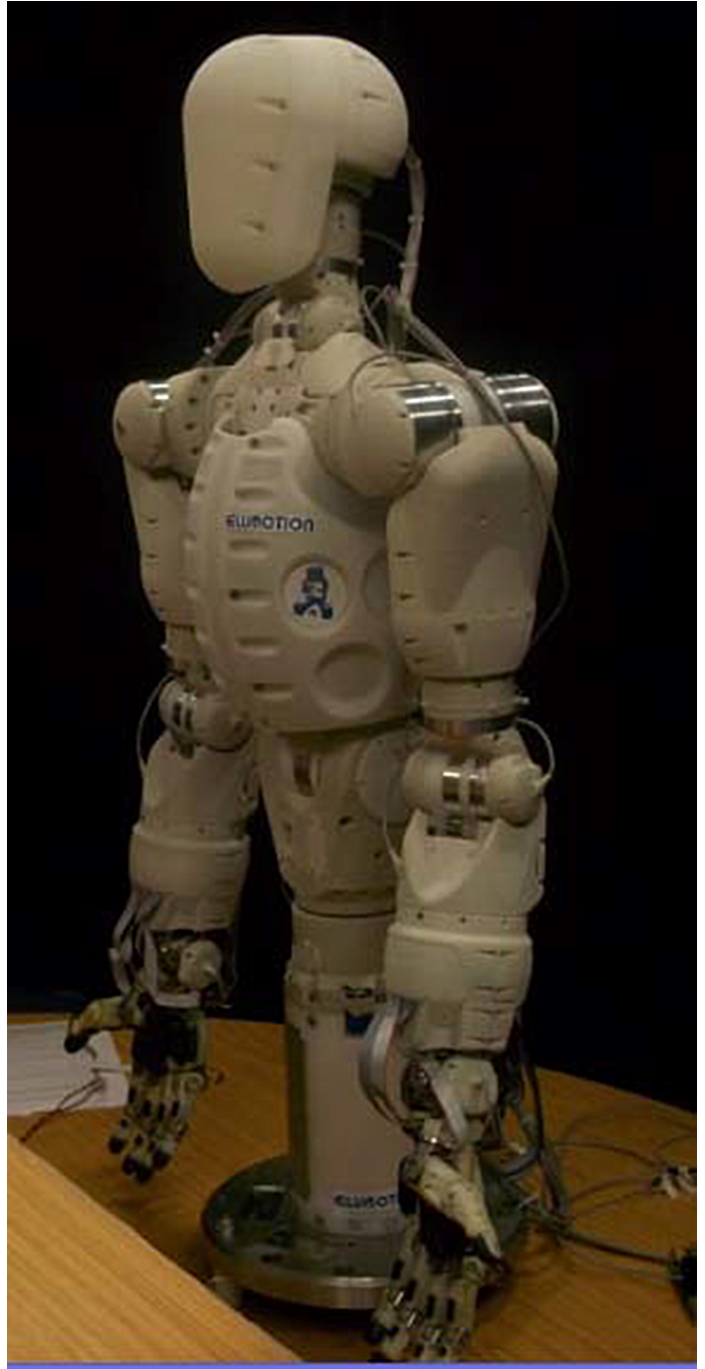

Figure 2. Bristol Robotics Laboratory BERT II robot.

\section{Integral sliding mode controller}

Integral slide mode (ISMC) control law (Shi et al., 2008) is presented here. The ISMC based task space (Cartesian) controller is briefly explained here and experimentally tested on the Bristol Robotics Laboratory's robot arm. The integral sliding mode variable $r$ is defined as follows (Herrmann et al., 2014):

$r=\dot{e}+K_{r} \boldsymbol{e}+K_{i} \int_{0}^{t} \boldsymbol{e}(\xi) d \xi-\dot{\boldsymbol{e}}(0)-K_{r} \boldsymbol{e}(0)$.

The position error vector is given by $\boldsymbol{e}=X_{\mathrm{d}}-X$, where $X_{\mathrm{d}}=\left[x_{\mathrm{d}}, y_{\mathrm{d}}\right]^{T}$ the Cartesian demand position (as we are controlling $x$ and $y$ only). The vectors $\dot{\boldsymbol{e}}(0)$ and $\boldsymbol{e}(0)$ are the initial values of the Cartesian velocity and position error respectively. It should be noted that the values of $K_{r}$ and $K_{i}$ define the desired behaviour of the control scheme once sliding mo-
Table 1. Physical Properties of BERT II arm.

\begin{tabular}{lllc}
\hline \multicolumn{3}{c}{ BERT II arm for 4 DOF, Physical Properties } \\
\hline Link & Mass (Kg) & Length (m) & Radius (m) \\
\hline 1 & $M_{1}=4$ & $L_{1}=0.21$ & $R_{1}=0.03$ \\
2 & $M_{2}=1.5$ & $L_{2}=0.23$ & $R_{2}=0.02$ \\
3 & $M_{3}=0.85$ & $L_{3}=0.23$ & $R_{3}=0.02$ \\
4 & $M_{4}=1.8$ & $L_{4}=0.23$ & $R_{4}=0.02$ \\
\hline
\end{tabular}

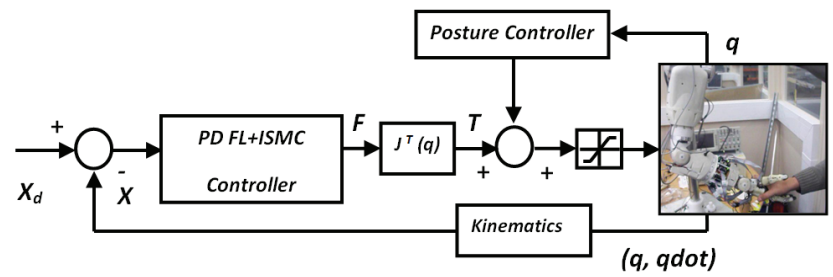

Figure 3. ISMC scheme.

tion $r=0$, is achieved. Hence, it acts like a reference model for the control scheme. The matrices $\mathbf{K}_{r}$ and $\mathbf{K}_{i}$ in this case are diagonal $(2 \times 2)$ and having positive scalar values.

As mentioned earlier, ISMC approach has an advantage in relation to other SMC methods (Shi et al., 2008; Utkin and Shi, 1996). From Eq. (1), it is easily seen that if the correct initial values, i.e. $\dot{\boldsymbol{e}}(0)$ and $\boldsymbol{e}(0)$, are used then $r(t=0)=0$. This is important, as sliding motion is secured from the start and aggressive controller action is kept minimal.

The general structure of the robot dynamics is given by:

$\Lambda(q) \ddot{q}+\mu(q, \dot{q})+v(q)=\tau$

where $\Lambda \in R e^{n \times n}$ is the inertia matrix, $\mu \in R e^{n \times 1}$ is the coriolis/centripetal vector. $v \in R e^{n \times 1}$ is the gravity vector. $\tau$ is the input torque. The $\Lambda(q), \mu(q, \dot{q})$ and $v(q)$ of the dynamic model (Lewis et al., 2003) for the BERT II arm has been obtained with the help of MapleSim (Khan, 2012; Maplesoft, 2015; Khan et al., 2010b) (an associated software package of Maple). Physical parameters of the robot are listed in Table 1.

The Cartesian space dynamics are now given as follows: Instead of joint torques, the dynamics consider the forces acting on the end effector:

$A(q) \ddot{X}+\mu_{c c}(q, \dot{q})+g(q)=f$

where $A=\left(J \Lambda^{-1} J^{T}\right)^{-1}, \quad \mu_{c c}=\bar{J}^{T} \mu-A \dot{J} \dot{q}, g=\bar{J}^{T} v$, $f=\bar{J}^{T} \tau$ and $X$ is the robot end-effector Cartesian position $\left(X=[x, y]^{T}\right)$, and $J$ is the Jacobian $\left(J=\frac{\delta X}{\delta q}\right)$. Hence, the Cartesian velocities are defined as $\dot{X}=J \dot{q}$. The matrix $\overline{\mathbf{J}}$ is the inertia weighted pseudo Jacobian inverse (Khatib, 1987; Nemec and Zlajpah, 2000): 
$\overline{\mathbf{J}}=\lambda^{-1} J^{T}\left(J \lambda^{-1} J^{\tau}\right)^{-1}$

The Cartesian position ( $x$ and $y$ ) of the end effector is used here to define the motion task of the robot. The combination of feedback linearization and ISMC Cartesian/task control law is:

$$
\begin{aligned}
F & =A \ddot{X}_{\mathrm{d}}+A K_{\mathrm{p}} e+A K_{\mathrm{d}} \dot{e}+g(q)+\mu_{c c}(q, \dot{q}) \\
& +\alpha \frac{r}{(\|r\|+\zeta)} .
\end{aligned}
$$

The scalars $K_{\mathrm{p}}$ and $K_{\mathrm{d}}$ are the proportional and the derivative gains respectively. The scalar $\alpha>0$ is normally selected large enough to reduce the effect of uncertainty and achieve robustness. The scalar $\zeta$, is introduced to minimize chattering effect (Jalani et al., 2010a). It should be noted that we are controlling only $x$ and $y$. Hence, we have two redundant degrees of freedom. These redundant degrees of freedom are used to produce human like motion employing the posture controller presented in the next section.

\subsection{Posture torque controller}

As mentioned in the previous section, the ISMC scheme is applied to a multi-redundant system (i.e. 4 DOF, shoulder flexion, shoulder abduction, humeral rotation and elbow flexion are used, while, only $2 \operatorname{DOF}(x$ and $y$ ) of the end-effector are controlled. Hence, the motion is underconstrained. Hence, a posture torque controller is employed which deals with the redundant motion, to produce human like posture. This posture controller also gives some sense of safety to the human interacting with the robot by generating human-like motion. This human like motion is achieved by minimizing an effort function (based on gravity) during reaching a particular point in the work space of the robot arm. The method here is adopted from the previous work by Spiers et al. (2009a, b) (see also De Sapio et al., 2005). The detailed description of the "posture" controller scheme along with experimental results can be found in Spiers et al. (2009a, b) and Khan et al. (2010a, b). The "posture" controller $\tau_{\mathrm{p}}$ is in the null space of the main controller given by Eq. (5) (Cartesian controller i.e. FL + ISMC); hence, it does not affect the main controller:

$\tau=J^{T} F+N^{T} \tau_{\mathrm{p}}, N^{T}=\left(\mathbf{I}-J^{T} \bar{J}^{T}\right)$

where $\mathbf{I}$ is the identity matrix. $\bar{J}$ is the the inertia weighted pseudo Jacobian inverse:

$\bar{J}=\lambda^{-1} J^{T}\left(J \lambda^{-1} J^{T}\right)^{-1}$

where $\lambda$ is the inertia matrix given by Eq. (2). The posture torque, $\tau_{\mathrm{p}}$ is defined as:

$\tau_{\mathrm{p}}=-K_{\mathrm{pp}} \frac{\delta U}{\delta q}-K_{\mathrm{dp}} \dot{q}$ where, $K_{\mathrm{pp}}$ and $K_{\mathrm{dp}}$ are proportional and derivative gains respectively. $U$ is the effort function defined as:

$U=\boldsymbol{v}^{T}\left(K_{\epsilon}\right)^{-1} \boldsymbol{v}$

and $v$ is the gravitational vector term from Eq. (2), and $K_{\epsilon}$ has $n \times n$ dimensions in our case, actuator activation diagonal matrix (having positive diagonal elements). The diagonal values $K_{\epsilon i}$, define the relative preferential weighting of each actuator $(i=1 \ldots n)$.

To get acceptable posture control performance, a good model of the inertia matrix $\lambda$ is required (in order to calculate Jacobian inverse given by Eq. 7).

The Cartesian control law (ISMC + FL) Eq. (5) is required for a better tracking accuracy. The control scheme is shown in Fig. 3 and the applied torques to the robot joints are given by $\tau=J^{T} F+\left(I-J^{T} \bar{J}^{T}\right) \tau_{\mathrm{p}}$

\subsection{ISMC Cartesian controller results discussion}

In this section experimental results are included for ISMC based tracking controller for 4 DOF robot arm. Also, simulation is included for the model reference compliance control scheme in Sect. "Model reference compliance control simulation using ISMC".

For the real time implementation of the control scheme, a dSPACE DS1106 embedded system is employed. A sampling time of $1 \mathrm{~ms}$ is used. The BERT II robot arm uses optical encoders for position and velocity measurements (see Fig. 4). As mentioned before, four degrees of freedom namely, shoulder flexion, shoulder abduction, humeral rotation and elbow flexion of the BERT II robot arm (Fig. 2) are used. The end-effector position is specified with respect to the fixed coordinate frame in the shoulder of the BERT II arm (see Fig. 4).

ISMC controller tuning is simple. Choosing suitable value for $\alpha>0$ large enough to reduce the effect of uncertainty and achieve robustness. The scalar $\zeta$, is selected to minimize the chattering effect (Jalani et al., 2010a; Herrmann et al., 2014).

Figures 5-6 show experimental results for ISMC based task space controller. In the real robot experiment 1 (Fig. 5), a multi-step demand is applied in the $x$ direction while a constant demand is applied in the $y$ direction. Tracking error is also shown which is very small. The control tuning parameters for experiment 1 are: $K_{\mathrm{p}}=2000, K_{\mathrm{d}}=10$, $K_{r 1}=K_{r 2}=8, K_{i 1}=K_{i 2}=16, \alpha=50$ and $\zeta=5$.

In another real experiment (Fig. 6), a sine wave demand is applied in $x$ and a constant demand is applied in the $y$ direction. The tracking error shown in Fig. 6 for experiment 2. Efficient real-time tracking results demonstrate the effectiveness of the proposed control scheme for our BERT II robot arm. The following control tuning parameters are used for experiment 2: $K_{\mathrm{p}}=2500, K_{\mathrm{d}}=10, K_{r 1}=K_{r 2}=8$, $K_{i 1}=K_{i 2}=16, \alpha=50$ and $\zeta=5$.

The scheme is effectively dealing with the deficiencies and uncertainties in the dynamic model. The ISMC has almost 


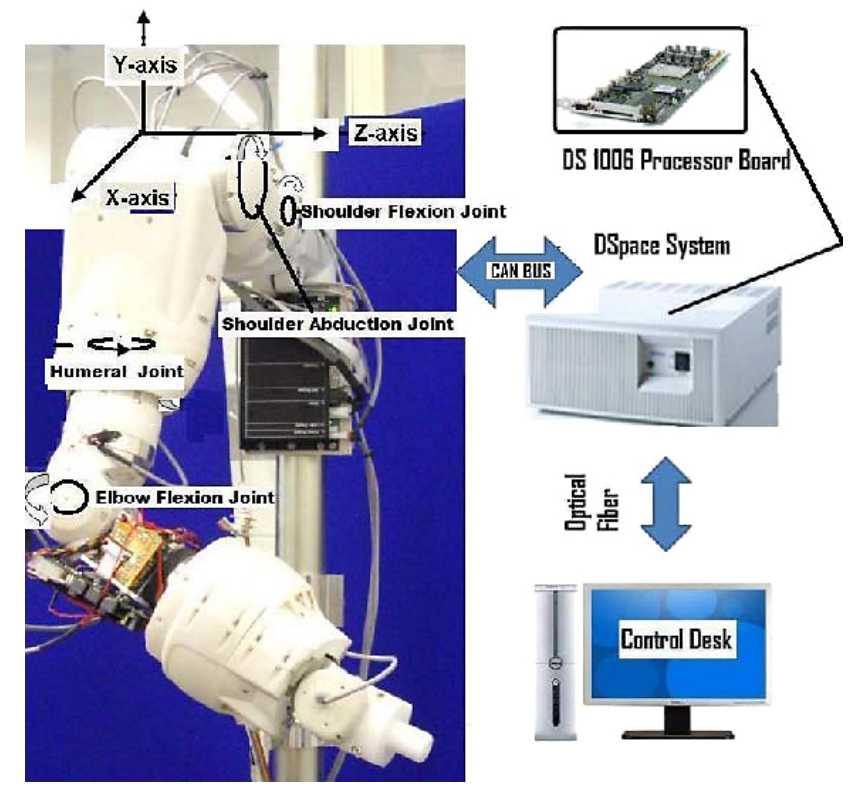

Figure 4. Experimental setup using dSPACE.

eliminated the chattering effect which normally would occur with traditional sliding mode schemes. The real advantage of this scheme here is to enhance the feedback linearization controller (using the 4 DOF dynamic model of the BERT II robot arm) with the ISMC to overcome the nonlinearities and unmodelled dynamics.

\section{Model reference compliance control using ISMC}

In this section, an ISMC based model reference compliance controller scheme is presented. This compliance scheme is similar in nature to the model reference adaptive compliance presented in (Khan et al., 2010b, 2014b), Khan and Herrmann (2014) and Colbaugh et al. (1996) However, here this scheme is much simpler and solely based on ISMC. The reference model is inside the sliding mode variable $r$. While in Khan et al. (2010b, 2014b), Khan and Herrmann (2014) and Colbaugh et al. (1996) the reference model is "external" where, tracking demand is modified (using force feedback) to compensate the external forces. The sliding mode element $r$ given by Eq. (1) is modified to introduce the compliance effect based on the external sensed force via joint torque sensors (Jalani et al., 2013; Herrmann et al., 2014):

$r=\dot{e}+K_{r} e+K_{i} \int_{0}^{t} e(\xi) d \xi-\int_{0}^{t} G_{f} H-\dot{e}(0)-K_{s} e(0)$

where, $G_{f}$ is a positive scalar and $H$ is the externally sensed force via joint torque sensors. The $K_{r}$ is the damping coefficient here and $K_{i}$ is the spring constant. The values of spring constant and damping coefficient can be used to get suitable level of compliance.
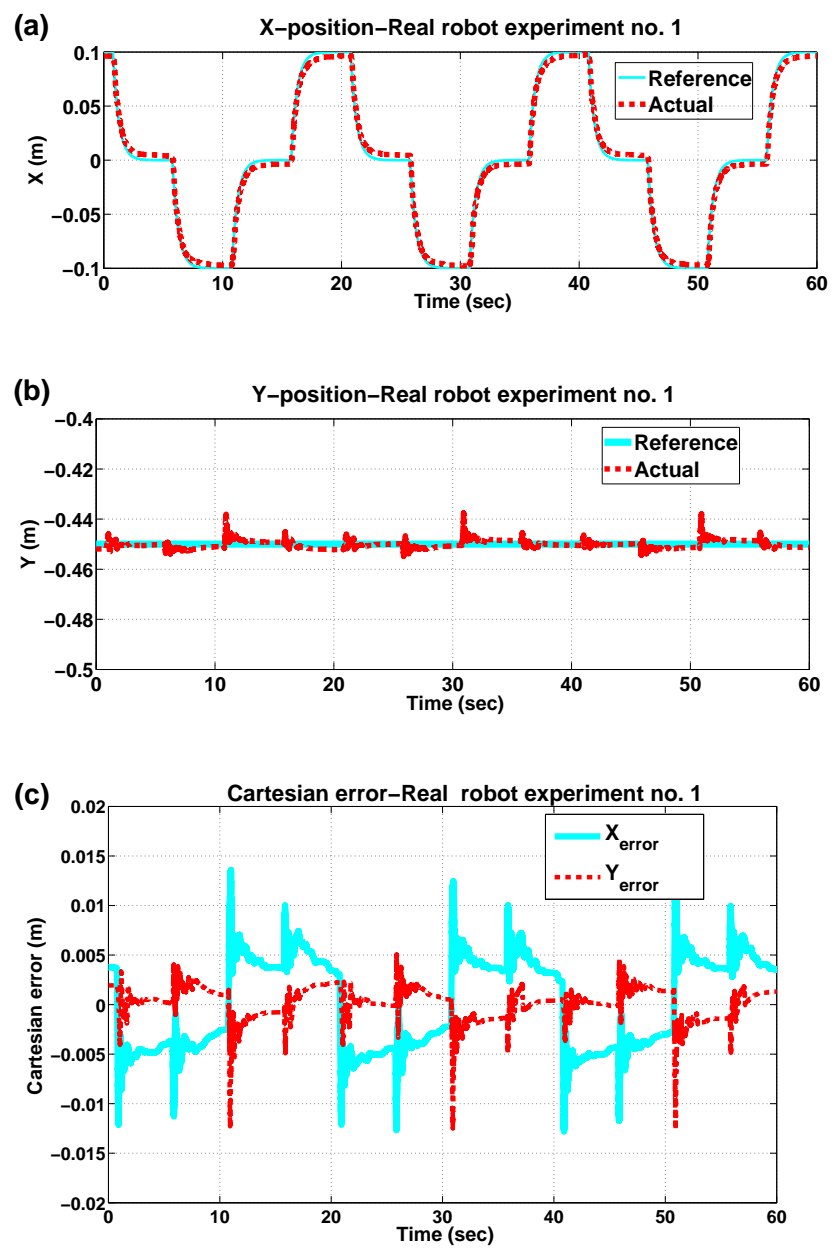

Figure 5. Position $x$ (a) and $y$ (b) and Position errors (c) experiment 1).

If we consider the derivative of sliding mode element $s$ in Eq. (10) (Jalani et al., 2013):

$\dot{r}=\ddot{e}+K_{r} \dot{e}+K_{i} e-G_{f} H$.

When the sliding mode is achieved i.e. $r=0$ and $\dot{r}=0$, then Eq. (11) becomes:

$\ddot{e}+K_{r} \dot{e}+K_{i} e=G_{f} H$.

Hence, the Cartesian error dynamics reduces to a second order mass-spring-damper system. The compliance behaviour of this reference model can be tuned by the selection of $K_{r}, K_{i}$ and $G_{f}$.

\section{Model reference compliance control simulation using ISMC}

In this section a compliance control scheme realised via ISMC is simulated on the BERT II arm. The is a similar Cartesian space $(x, y$ and $z$ ) scheme to the one presented and 


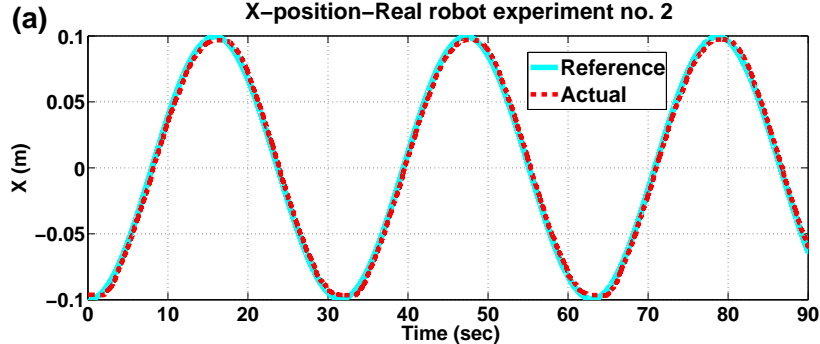

(b) Y-position-Real robot experiment no. 2
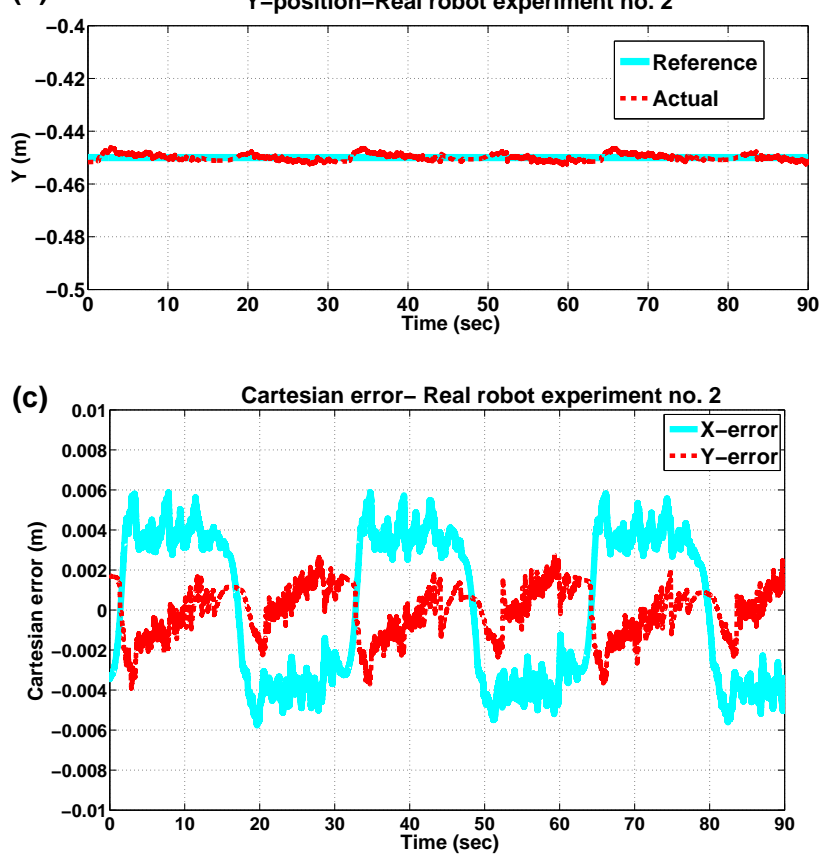

Figure 6. Position $x$ (a) and $y$ (b) and Position errors (c) experiment 2).

experimental result were presented in Sect. 2. However, here is focus on compliant behaviour. Figure 7 shows the compliance simulation results. External forces of $5 \mathrm{~N}$ are applied on $x$ and $z$ direction while $K_{r}=8 \mathrm{~N} \mathrm{~s} \mathrm{~m}^{-1}$ and $K_{i}=10 \mathrm{~N} \mathrm{~m}^{-1}$. In the absence of external forces, the controller will track the reference position demands (in this case a constant demand for $x, y$ and $z$ ). In the presence of external forces robot will follow a modified trajectory $\left(x_{\mathrm{d}}, y_{\mathrm{d}}\right.$ and $\left.z_{\mathrm{d}}\right)$ to compensate for the forces and behave compliantly. The compliance level is created by choosing the values $K_{r}$ and $K_{i}$. Similarly, in Fig. 8, another set of compliance results are shown. The scheme can be made more compliant by selecting lower value for $K_{r}$. It should be noted that joint torques sensors/end effector force sensor are essential for real implementation of this controller (Herrmann et al., 2014). It is worth pointing out that decoupling external torques from robot body-own torques may be challenging. However, we have addressed this issue to some extent in our previous work (Khan et al., 2014b, 2010a). It is to be noted that the geometric split-

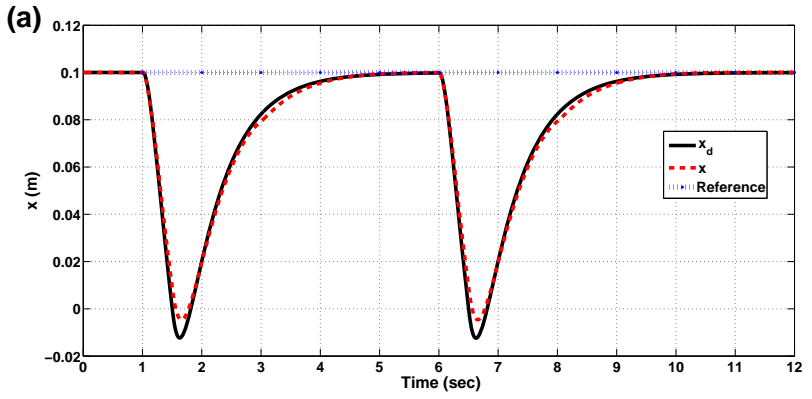

(b)

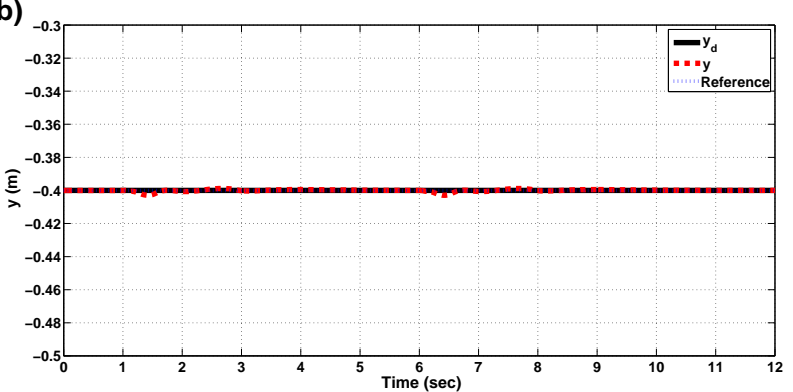

(c)

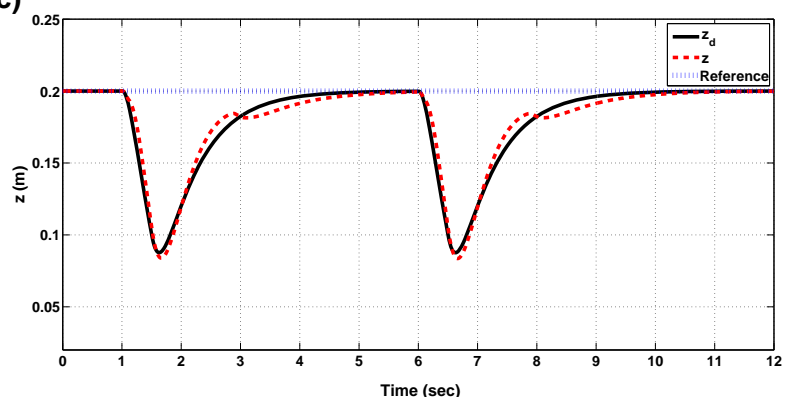

Figure 7. Cartesian position $x$ (a) and $y$ (b) and $z$ (c) for $K_{r}=8 \mathrm{~N} \mathrm{~s} \mathrm{~m}^{-1}$ and $K_{i}=10 \mathrm{~N} \mathrm{~m}^{-1}(F x=F z=5 \mathrm{~N}$ and $F y=0)$.

ting (i.e. decoupling torques) of the control task of the endeffector and the control of the redundant degrees of freedom has been introduced by (De Sapio et al., 2005). The idea is that multiple configurations were avoided by introducing and minimizing cost which resolves the redundancy issues. Other applications by using decoupling technique can be found in Herrmann et al. (2014) and Spiers et al. (2009a, b).

\section{Conclusions}

Safe physical human robot interaction lies in the core of social robotics. Safety needs multi-dimensional approach and compliance alone cannot solve safety problem. However, compliance can play an important role in providing immediate layer of safety. In this paper, compliance control has been realised through a model reference integral sliding mode control. An ISMC tracking control scheme is turned into a model reference compliance controller, by introducing external force feedback into the sliding mode element. The de- 
(a)

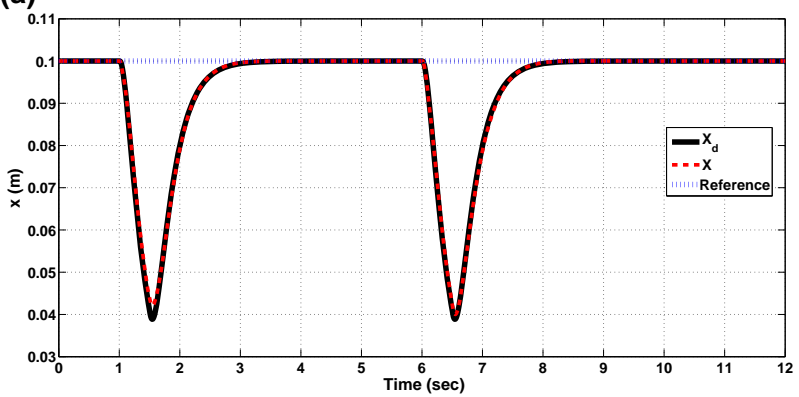

(b)

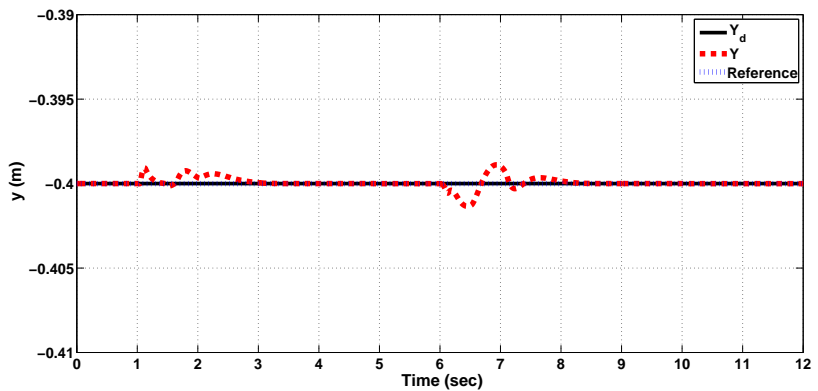

(c)

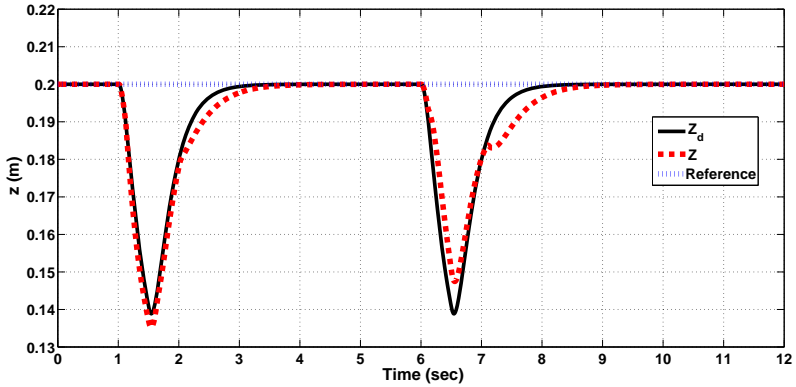

Figure 8. Cartesian position $x$ (a) and $y$ (b) and $z$ (c) for $K_{r}=12 \mathrm{~N} \mathrm{~s} \mathrm{~m}^{-1}$ and $K_{i}=30 \mathrm{Nm}^{-1} \quad(F x=F z=5 \mathrm{~N}$ and $F y=0)$.

sired compliance level can be achieved simply by changing the derivative and integral gain in the sliding mode variable $r$. ISMC is simple and very easy to implement. The tuning of the control scheme for the desired performance is also very straightforward as there are not many parameters to be tuned. Real-time Cartesian position tracking results and compliance control simulation included here highlights the flexibility of ISMC based control and its effectiveness to be used as model reference compliance control scheme. The ultimate goal of this work is to use the ISMC based control scheme for safe human-robot interaction.

Acknowledgements. Both the authors would like to thank Bristol Robotics Laboratory (BRL) UK for the great support they got during their $\mathrm{PhD}$ studies. The experimental results presented here were produced in BRL, UK.
Edited by: X. Ding Reviewed by: three anonymous referees

\section{References}

Ajwad, S. A., Ullah, M. I., Baizid, K., and Iqbal, J.: A comprehensive state-of-the-art on control of industrial articulated robots, J. Balkan Tribolog. Assoc., 20, 1310-4772, 2014.

Ajwad, S. A., Iqbal, J., Ullah, M. I., and Mehmood, A.: A systematic review of current and emergent manipulator control approaches, in: Frontiers of Mechanical Engineering, Springer, Berlin, Heidelberg, Germany, 198-210, 2015.

Braun, D., Petit, F., Huber, F., Haddadin, S., van der Smagt, P., Albu-Schäffer, A., and Vijayakumar, S.: Robots Driven by Compliant Actuators, IEEE T. Opt. Control Actuat. Constr. Robot., 29, 1085-1101, 2013.

Castaños, F. and Fridman, L.: Analysis and Design of Integral Sliding Manifolds for Systems With Unmatched Perturbations, IEEE T. Autom. Contr., 51, 853-858, 2006.

Colbaugh, R., Glass, K., and Wedeward, K.: Adaptive Compliance Control of Electrically-Driven Manipulators, in: Proceedings of the 35th Conference on Decision and Control, Kobe, Japan, 394 399, 1996.

De Sapio, V., Khatib, O., and Delp, S.: Simulating the task level control of human motion: a methodology and framework for implementation, Visual Comput., 21, 289-302, 2005.

Di Natali, C. and Valdastri, P.: Surgical robotics and instrumentation, Surgery, 245, 379-384, 2012.

Ding, X. and Fang, C.: A Novel Method of Motion Planning for an Anthropomorphic Arm Based on Movement Primitives, IEEE/ASME T. Mechatron., 18, 624-636, doi:10.1109/TMECH.2012.2197405, 2013.

Eker, I. and Akinal, S.: Sliding mode control with integral augmented sliding surface: design and experimental application to an electromechanical system, Elect. Eng., 90, 189-197, 2008.

Ficuciello, F. and Villani, L.: Compliant hand-arm control with soft fingers and force sensing for human-robot interaction, in: Biomedical Robotics and Biomechatronics (BioRob), 2012 4th IEEE RAS \& EMBS International Conference Rome, Italy, 1961-1966, 2012.

Herrmann, G., Jalani, J., Mahyuddin, M. N., Khan, S. G., and Melhuish, C.: Robotic hand posture and compliant grasping control using operational space and integral sliding mode control, Robotica, 1-23, doi:10.1017/S0263574714002811, 2014.

Iqbal, U., Samad, A., Nissa, Z., and Iqbal, J.: Embedded control system for AUTAREP - A novel AUTonomous Articulated Robotic Educational Platform, Tehnicki Vjesnik-Technical Gazette, 21, 1255-1261, 2014.

Islam, R. U., Iqbal, J., and Khan, Q.: Design and comparison of two control strategies for multi-DOF articulated robotic arm manipulator, in: vol. 02, Control Engineering and Applied Informatics (CEAI), Bucharest, Romania, 1454-8658, 2014.

Jalani, J., Herrmann, G., and Melhuish, C.: Robust Trajectory Following for Underactuated Robot Fingers, in: UKACC International Conference on CONTROL, Conventry, UK, 2010a.

Jalani, J., Herrmann, G., and Melhuish, C.: Concept for Robust Compliance Control of Robot Fingers, in: 11th Conference Towards Autonomous Robotic Systems, Plymouth, UK, 97-102, 2010b. 
Jalani, J., Mahyuddin, N., Herrmann, G., and Melhuish, C.: Active robot hand compliance using operational space and Integral Sliding Mode Control, in: vol. 01, IEEE/ASME International Conference on Advanced Intelligent Mechatronics (AIM), Wollongong, NSW Australia, 1749-1754, 2013.

Khan, S.: Adaptive and reinforcement learning control methods for active compliance control of a humanoid robot arm, $\mathrm{PhD}$ Thesis, University of the West of England, Bristol, UK, 2012.

Khan, S. and Herrmann, G.: NRCGHRIComplianceControl, https:// www.youtube.com/watch?v=IKE8Rrtr-Ow (last access: 11 January 2016), 2014.

Khan, S., Herrmann, G., Pipe, T., and Melhuish, C.: Adaptive multidimensional compliance control of a humanoid robotic arm with anti-windup compensation, in: IEEE/RSJ International Conference on Intelligent Robots and Systems (IROS), 2218-2223, 2010a.

Khan, S., Herrmann, G., Pipe, T., Melhuish, C., and Spiers, A.: Safe Adaptive Compliance Control of a Humanoid Robotic Arm with Anti-Windup Compensation and Posture Control, Int. J. Social Robot., 2, 305-319, 2010b.

Khan, S. G., Herrmann, G., Al Grafi, M., Pipe, T., and Melhuish, C.: Compliance Control and Human Robot Interaction Part I Survey, Int. J. Human. Robot., 11, 1430001-1-1430001-28, 2014a.

Khan, S. G., Herrmann, G., Lenz, A., Al Grafi, M., Pipe, T., and Melhuish, C.: Compliance Control and Human Robot Interaction Part II Experimental Examples, Int. J. Human. Robot., 11, 1430002-1-430002-21, 2014b.

Khatib, O.: A Unified Approach for Motion and Force Control of Robot Manipulators: The Operational Space Formulation, IEEE J. Robot. Autom., RA3, 43-53, 1987.

Kim, H., Kim, I., Cho, C., and Song, J.: Safe joint module for safe robot arm based on passive and active compliance method, Mechatronics, 22, 1023-1030, 2012.

Lewis, F., Dawson, D., and Abdallah, C.: Robot Manipulator Control: Theory and Practice, Marcel Dekker Inc., New York, USA, 2003
Makoto, Y., Gyu-Nam, K., and Masahiko, T.: Integral Sliding Mode Control with Anti-windup Compensation and Its Application to a Power Assist System, J. Vibrat. Control, 16, 503-512, doi:10.1177/1077546309106143, 2010.

Maplesoft: MapleSim, http://www.maplesoft.com/products/ maplesim/modelgallery/detail.aspx?id=108 (last access: 11 January 2016), 2015.

Nemec, B. and Zlajpah, L.: Null space velocity control with dynamically consistent pseudo-inverse, Robotica, 18, 513-518, 2000.

Potkonjak, V., Jovanovic, K., Svetozarevic, B., Holland, O., and Mikicic, D.: Modeling and Control of a Compliantly Engineered Anthropomimetic Robot in Contact Tasks, in: 35th mechanisms and robotics conference, ASME, Portland, Oregon, USA, 2011.

Rezoug, A. B. T. and Hamerlain, M.: Experimental Study of Nonsingular Terminal Sliding Mode Controller for Robot Arm Actuated by Pneumatic Artificial Muscles, 19th World Congress The International Federation of Automatic Control (IFAC), 2429 August 2014, Cape Town, South Africa, 10113-10118, 2014.

Shi, J., Liu, H., and Bajcinca, N.: Robust control of robotic manipulators based on integral sliding mode, Int. J. Control, 81, 15371548, 2008.

Spiers, A., Herrmann, G., and Melhuish, C.: Implementing ?Discomfort? in Operational Space: Practical Application of a Human Motion Inspired Robot Controller, TAROS conference: Towards Autonomous Robotic Systems, Derry, Northern Ireland, UK, 2009a.

Spiers, A., Herrmann, G., Melhuish, C., Pipe, T., and Lenz, A.: Robotic Implementation of Realistic Reaching Motion using a Sliding Mode/Operational Space Controller, Lecture Notes in Computer Science (ICSR '09), Springer-Verlag, Berlin, Heidelberg, Germany, 230-238, 2009b.

Utkin, V. and Shi, J.: Integral sliding mode in systems operating under uncertainty conditions, IEEE Proceedings of the 35th Decision and Control, Kobe, Japan, 4591-4596, 1996. 